\title{
Exploring design options for interactive video with the Mnemovie hypervideo system
}

\begin{abstract}
Mnemovie is an investigative hypervideo system for exploring design options for interactivity with digital motion picture files (video). The custom-designed software toolset is used to build a series of experimental interactive models from which three models were subsequently developed for initial user experience testing and evaluation. We compared interaction with each of the models across three groups of video file users, from expert to non-expert. Understanding participants' preference for each model helps define the different dimensions of the actual user experience. We discuss how these findings and the subsequent development of persona scenarios can inform the design of hyper-video systems and the implications this has for interaction design.
\end{abstract}

\section{Keywords}

interaction design, digital video, evaluation, user behaviour, persona.

The problem with storing and retrieving digital video files using information and communication technologies that employ text-based indexing systems, is essentially one of semantics; from handycams to motion pictures shot on a mobile phone, the complexities of words do not serve well the complexities of retrieving the motion picture document. As Glorianna Davenport at MIT observed a decade ago: “..keyword matching is a crude and unsatisfactory method for sampling the information content of complex sources...." (Davenport, 1996. We address the paucity of design evident in popular video databases such as YouTube and GoogleMovies where the viewer has a television-like experience made up of selected individual movie items. These are assembled by guessing words used in metadata registration of 
uploaded material and interaction with the movie database, thereby following in the sequential tradition of analogue video and film.

Digital video however, enables access to individual frames of a motion picture recording, where in an interactive system, the participant is able to define duration and relational ordering of individual 'scenes' and shots. The potential is to recover information from motion pictures more efficiently than a reliance on text-based indexing, or to have the opportunity to interact creatively with the phenomena of the motion picture experience.

The objective of the Mnemovie research project is to propose more efficient and stimulating means to store and retrieve the video files with which we work, communicate and entertain ourselves, increasingly each year. The process of designing an interactive system is complex and needs to progress with evaluative feedback at early stages of conceptual design and prototyping.

The first author, from a film and video background, experienced with experimental and reflective practice in analogue and digital domains, collaborated in the development of an evaluation process with a cognitive scientist specialising in user-centred design (UCD). The collaboration was designed to provide detailed and specific responses from a group of volunteer participants to outcomes of the interactive design and development process.

In these studies, visual memory for people, places and objects are investigated as mnemonic devices to provide cues for navigating through a movie file collection. A series of experimental models evolved to three test versions to help us understand users' experience and the effectiveness of the system for completing set tasks. An evaluation study combining observation, questionnaires and semi-structured interviews, the core of this paper, captured the different dimensions of user experience. The findings provide useful feedback about design improvements for the Mnemovie interactive system and toolset assessing efficiency, engagement and creativity support for digital movie users. 


\section{Background}

The literature on machine memory and systems of indexing images using metadata - keyword descriptions of images - reveals both reluctance and eagerness by computer scientists to simplify options for engaging with collections of motion pictures and still photographs. Ballard and Brown (1982) described relational database models in the early 1980s as turning away from representing models, to matching models from within a knowledge base where proposition and inference became important aspects of interaction. These approaches have become central to scientific, medical and surveillance sorting, storage and retrieval systems, (Ballard and Brown, 1982). Research projects seeking industrial objectives for television, cable and broadband management of movies have included IBM Cue Video (Amir, 2000.), using a semi-automated word annotation system. By contrast, an image-based relational system, i-Map, enabled the visualisation and navigation of a large scale photography database by clustering magnified images onscreen, allowing the user to "...explore areas which look more promising...", through the selection of an initial image for which the system would then seek further matches, before re-clustering (Lim et al., 2004). Exploring areas that look more promising is the kind of query formulation favoured by researchers in pursuit of non-specific information or data. This and other research has informed the development of the Mnemovie system and led to research exploring the potential of 'hypervideo'. Important work achieved by the Hyper-Hitchcock project for instance, concentrated research effort on legibility in the system:

"Hypervideo, like hypertext, derives its value by allowing users to express their own information needs via navigation. But navigation is only valuable when the viewer can anticipate the outcome of navigation or assess it very rapidly." (Girgensohn et al., 2003). In our research, anticipation of the overall shape of interactive possibilities together with the scope of the video files collected into a hyper-video system is the framework within which the research has progressed. At the outset it was necessary to maintain the functional objectives of the investigation as dependent on high-level concepts that elevated qualities of experience and tacit knowledge as contributing to interaction as a creative reflexive response. 


\section{Mnemovie Hypervideo Model Design}

The Mnemovie system developed from experiments using practice-based research methods as distinct from a user-centred design exercise. This approach is similar to an art creation process, where the concept is developed through the practitioner's knowledge, experience, skills and sense of creative enquiry. The Mnemovie system was implemented as a software engine toolset in collaboration with a technical developer. The engine, in simplifying the coding of the system, enables the practioner as researcher to use the toolset for making experimental "beta" models. Through reflective analysis and iterative development of each model, an understanding of a user's experience takes shape. As presented in this study, components of user research came into the process for evaluation purposes after the prototype designs were developed. Our evaluation study provided the practitioner with an understanding of the user context for the Mnemovie models made using the toolset rather than validating whether requirements of a specific design were met.

The strategy for interaction with the experimental models used the mouse or the arrow keys on the computer keyboard to effect 4-way interaction: the $\uparrow$-key for forward playback of the movie, $\downarrow$-key for backward motion. The $\leftarrow$-key and $\rightarrow$-key launch movie files linked to visual cues (mnemonics) in the movie being viewed (Fig 1).

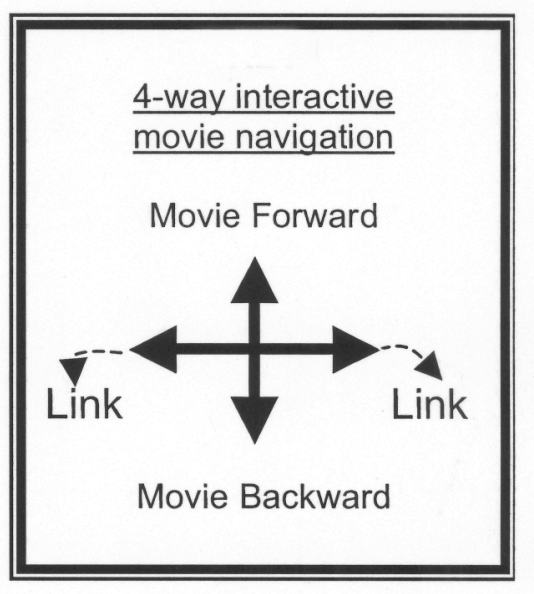

Figure 1: Mnemovie navigation schematic using computer keyboard arrow keys 
A series of initial experimental models were built using a robust and flexible software framework, the custom-made Mnemovie engine and toolset, to link using mnemonic cues visible within the picture of movie files encountered as full screen motion-picture images. Three test models were then evolved for evaluation purposes to help us understand users' experience and the effectiveness of the system for completing set tasks.

Each of the three test models use the same 4-way interaction approach (Fig 1) to enable links to be made with the same video file collection of short 3-minute documentaries (about the work of twelve HCI researchers). The interaction design of each model is characterised to the participant as a schema, described to the participant with a single word: CIRCLE; LINE; GRID. The reason each test model has a title, characterized by the name of a shape, (LINE, GRID, CIRCLE) is to help with understanding and remembering the navigational principles, or schema, of each one. This provides the participant with an initial shape describing the relation between the first video encountered and the collection of twelve videos with which links are made. In addition to the three test models, a practice model was used to familiarise participants with interaction principles, with a schema and collection of video files different to that used in the test models. The hypervideo conceptual schemas evaluated are outlined in the following user scenarios in which interaction follows the pattern of: navigating, linking, watching, and returning to navigation.

\subsection{CIRCLE Test Model}

Interaction with the CIRCLE test model commences with navigating the collection of videos. These appear as 'speeded-up' movement of the audio-visual content, achieved by sampling the original video to make a shorter 'skimmed' movie, the original 3-minute movie thereby being reduced to 3-4 seconds. All twelve movies when joined loop as a 36-second cycle. The 个-key runs the loop forward, the $\downarrow$-key runs it in reverse. 


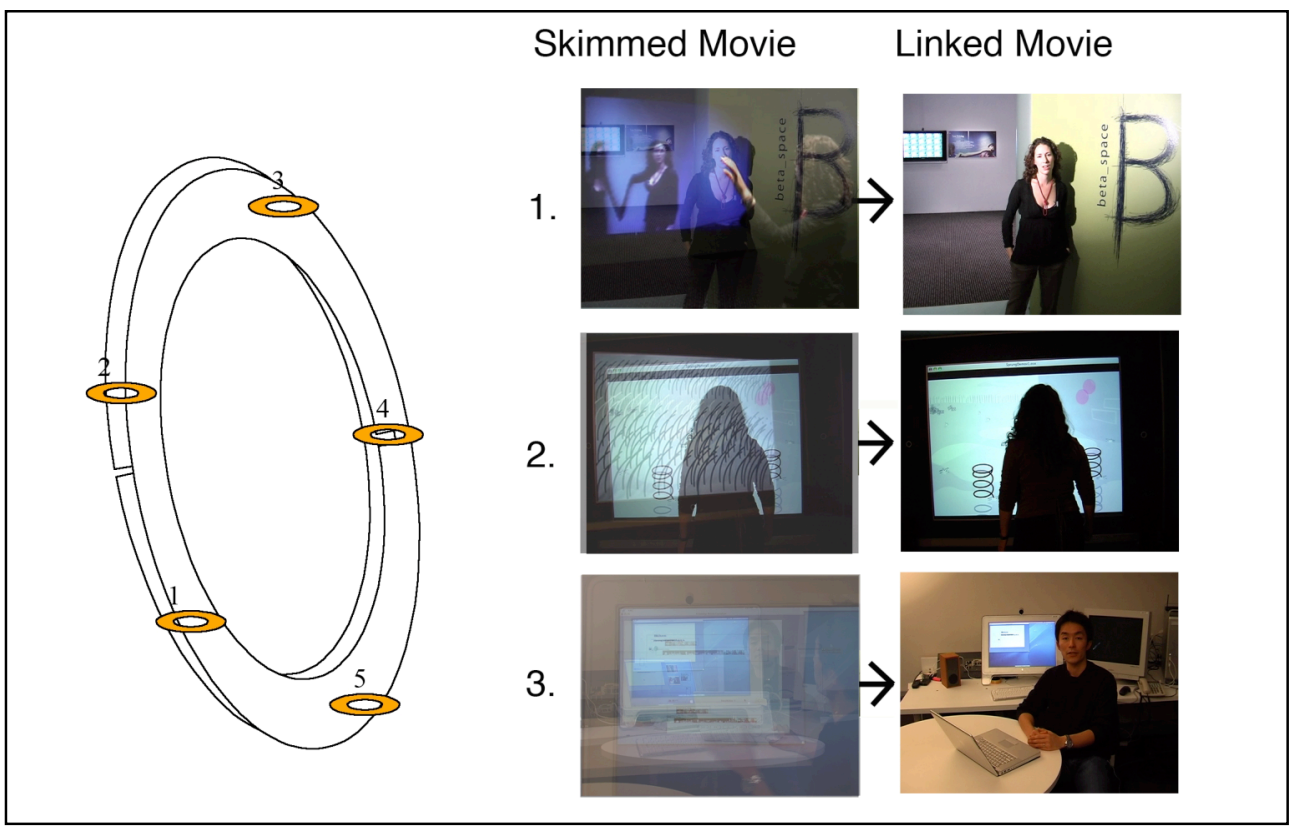

Fig 2: Test model 'CIRCLE' navigational schema. Each 'skimmed' movie is joined sequentially, the last linked to the first to make a durational loop (as in the diagram at left). Each 'skimmed' movie (centre) is linked to the full version (right) and accessed interactively.

Linking: is achieved by using the $\leftarrow$-key or the $\rightarrow$-key to link to one of the movies, relational to its indexical 'skimmed' version on the loop (Figure 2).

Returning: is achieved by using the $\leftarrow$-key again to exit to the previous entry point on the 'skimmed' loop movie; using the $\rightarrow$-key advances directly to the next movie in the collection, preceded with a title, the name and research topic of the video subject. In the CIRCLE model, the participant has a choice of retrieving and viewing a movie using either the glimpsed visual cues, the mnemonics of person or object, or the text-based titles preceding each movie.

\subsection{LINE Test Model}

Interaction with the LINE test model commences with a Point-of-View (POV) of the scene recorded by the camera, travelling from the beach, over dunes, along a road, through scrub bush, across a lake and into the rainforest (Figure 3). 


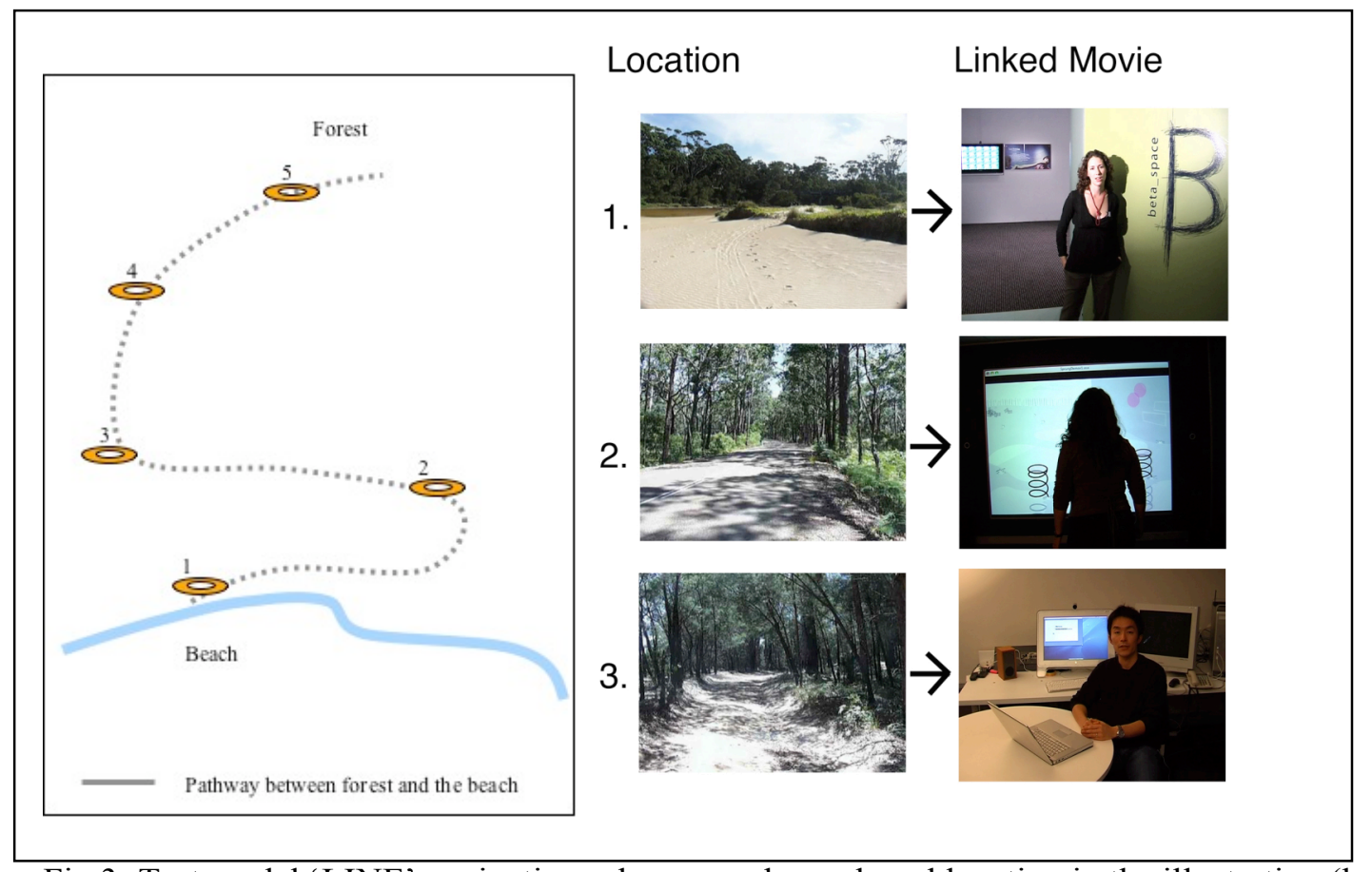

Fig 3: Test model 'LINE' navigation schema: each numbered location in the illustration (left)

refers to a specific Location in the POV movie, (centre), hyperlinked to a specific Linked Movie (right).

Navigating occurs from the beach to the forest where at any point the $\downarrow$-key will show the scene behind $\left(180^{\circ}\right)$ the previous POV, moving back toward the starting point. Thereby the user is able to 'move' up and down the pathway recorded in the movie.

Linking: the navigation track is roughly divided into twelve sections, each distinguished by landscape features that are the mnemonics - memory cues. The participant uses the $\leftarrow$-key or $\rightarrow-$ key to link to one of the twelve movies in the collection. For example, Location 1, 2 and 3 (Figure 3) are the 'places' (the mnemonic), with which a link is associated.

Returning: use of the $\leftarrow$-key again exits to the same point on the POV movie; using the $\rightarrow$-key advances directly to the next movie in the collection, with the title of the name and research topic of the subject. In the LINE model, the participant has a choice of accessing and viewing a movie using either the visual cues learnt during initial exploration, or the text-based titles preceding each of the movies. 


\subsection{GRID Test Model}

The GRID model employs the principles described above for LINE with the added feature of navigating a series of intercepting straight lines - streets - the grid structure of an inner city block (Figure 4). The map of the inner city grid of streets, as seen on the right, is the concept schema for locating each of the twelve movies divided around the streets, while the arrow shows the interactive route taken to navigate from Location 1 to 3.

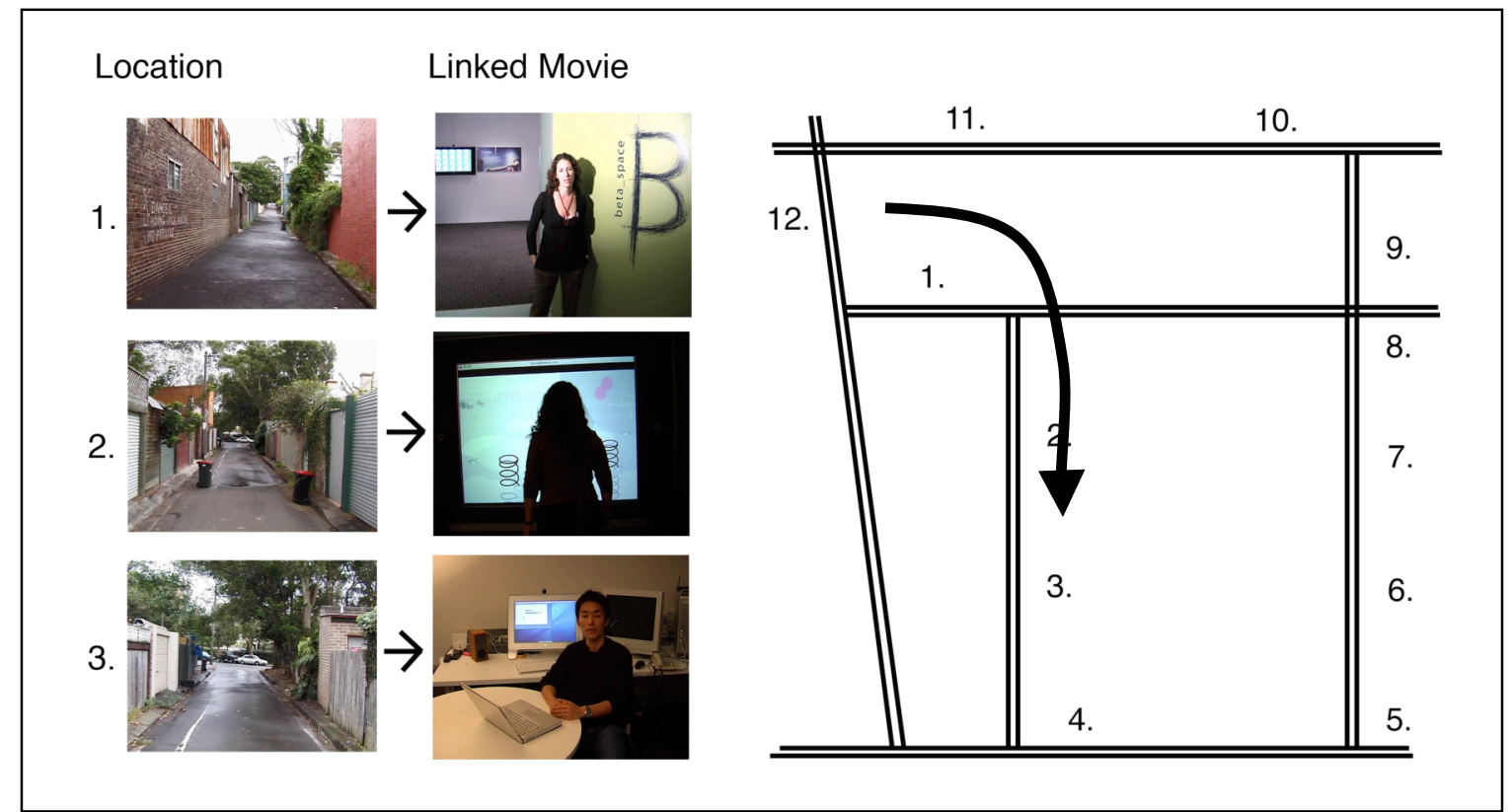

Fig 4: Test model, 'GRID' navigation schema: the Location frames 1, 2 and 3 are what the participant sees as a motion picture POV (left). Each specific Location is hyperlinked to a Linked Movie (centre). The street grid diagram (right), indicates the conceptual path taken.

Navigating: using the $\uparrow$-key runs the POV movie forward, the $\downarrow$-key turns the viewpoint through $180^{\circ}$ and returns.

Linking: using the $\leftarrow$-key or the $\rightarrow$-key will achieve one of two outcomes: a) when adjacent to a corner, link to the movie POV of the adjoining street; b) relational to its location on a section of the street, link to one of the twelve movies. For example, location 1, 2 and 3 (Figure 4) are the 'places' with which a link is associated.

Returning: using the $\leftarrow$-key again exits to the same point on the POV movie; using the $\rightarrow$-key advances directly to the next movie in the collection, with the title of the name and research 
topic of the subject. In the GRID model, the participant has a choice of accessing and viewing a movie using either the visual cues learnt during initial exploration, or the text-based title.

\section{User Experience Evaluation Study}

In developing an evaluation process designed to provide detailed and specific responses to this stage of the design process, several approaches were taken for combining together research paradigms and techniques established in recent years (Candy, 2007, Bilda, 2006) (Karat, 1994, Leritz-Higgins, 2004, Preece et al., 2002). In this section, we describe in detail each step (1-6) taken in the process of gathering the data from the interactive encounters. In the following two sections, we describe the results and the analysis and how this informs the design options for further development of the Mnemovie system.

\subsection{Planning}

The evaluation objectives were to inform the formative process of development, ("inform" in its root meaning of "shape", or 'affecting with a feeling, imbuing a spirit'). In the preparation for the study we explored a number of research issues regarding the qualities of the Mnemovie interactive experience: for example, effectiveness for navigating and retrieving movies from the collection; the effect or impact of user expertise level on the experience and evaluation of the Mnemovie test models; any differences in efficiency and effectiveness between the test models. To address these questions we employed an evaluation plan commencing with the recruitment of participants using pre-determined participant profiles. (A pilot test of the entire plan was conducted with a colleague, proceeding as if were the real thing, before recruiting volunteer participants). Preliminary user information was gathered with questionnaires before training participants to use the interactive models employing a practice model. Specific tasks were set following participant exploration and experience with each of the three test models in turn. A final questionnaire about the experience and an interview session was followed by presentation and analysis of results. 


\subsection{Selecting and determining participant profiles}

The group of thirteen participants was recruited using flyers distributed amongst the community of computer users in Sydney who access movie files for a variety of purposes. Participant profiles were gathered from amongst forms submitted by volunteers, divided between a mix ranging from expert to non-expert movie users, and near equal gender dispersal. The participant completed an initial questionnaire to enable us to establish individual profiles: we asked participants how often they encountered motion pictures, (as cinema, television, online etc.), how often, which different locations and social contexts. Using data gathered about the frequency with which they used movie files on computers, we classified participants as a movie expert, a movie-maker and a movie-user. As the goal of the evaluation concerns a computerbased system, people who would not normally use a computer were excluded from the testing.

\subsection{Practice session}

In the practice model the movie collection encountered is different from that used in the three test models. Following written and spoken instruction participants are asked to interact with the practice model that concerned the contents of a chest of drawers. This includes the task of retrieving a specific movie file linked to one of the objects in one of the drawers. Participants are told that 'remembering where you 'find' the movies within the navigational schema, is important for being able to complete the tasks'. The practice model is designed to be interactively reasonably complex, to 'skill-up' the participant for the test models ahead.

\subsection{Testing Sessions}

The sessions were conducted in a medium-sized, quiet office-type room, with the researcher sitting alongside the participant. Each model is launched from the computer desktop, a plain white window with the icon title (PRACTICE, GRID, LINE or CIRCLE). Each title is intended to help with understanding and remembering the navigational principles, or schema, of each one, characterized by the name of a shape, LINE, GRID, CIRCLE. These iconic titles enable the ordering of each model in the final questionnaire. The testing procedure for each model is read 
out loud by the researcher, (or if the participant prefers, themselves reading), together with the description of its interactive principles. Each participant encounters the three models in a different order from other participants.

\subsection{Procedure for a Testing Session}

1. The participant is asked to repeat the operating principles to the researcher before launching the specified named model from the desktop.

2. During the exploration phase that follows, participants often discover the two different methods of exiting the 3-minute movie, either returning to the initial movie location or to the text-based titles. These two forms of 'indexing' the movies for later retrieval are central to the two retrieval tasks set after the participant has become familiar with the navigation of the model.

3. Following 3-5-minutes of exploration and play with the model, the participant completes two retrieval tasks. The first movie to be retrieved is one that has previously been seen by the participant during the exploration period; the second task is to retrieve a movie that the participant has not seen. The researcher describes only the movie content when instructing the participant to retrieve a movie file. For example: 'Find the movie about the man working with the trombone' or 'Find the movie about the woman who sits against a red background'.

4. Having completed the retrieval task the participant repeats the process for the remaining test models.

5. The data gathered through these observations are augmented with data supplied by each participant through the final questionnaire and interview.

\subsection{Final questionnaire and interviewing}

The final stage addresses user-perceived evaluation of three models and is conducted in three sections. The first section, in questionnaire format, requests the participant to rank several characteristics of the three test models using a matrix table, placing the title of each model in the 
column orders High, Moderate and Low, in response to the following criteria: ease of use; opportunity for explorative interactivity; efficiency of search function; quality of the experience and the overall preference.

In the second section, the ranking outcomes are compared with participants' viewpoints about the interactive principles of the three models using a keyword, or short conceptual sentence, together with the strongest and the weakest features encountered.

The third section is a semi-structured interview opening with a discussion about movie collections and the kind of movie or cinema experience the participant usually encounters. Then the researcher asks for a description of the kind of movies or movie collection for which the participant could use this navigational approach, or the kind of movie collection they would like to assemble to be able to use this navigational approach.

\section{Data Results}

The ranking data from the second questionnaire counted the participants' preferences - High, Moderate, Low - for each of the models. The results show (fig 5) the CIRCLE model quite clearly scored High for Ease of Use and Efficiency in retrieving target movies, and LINE consistently follows as a Moderate alternative.
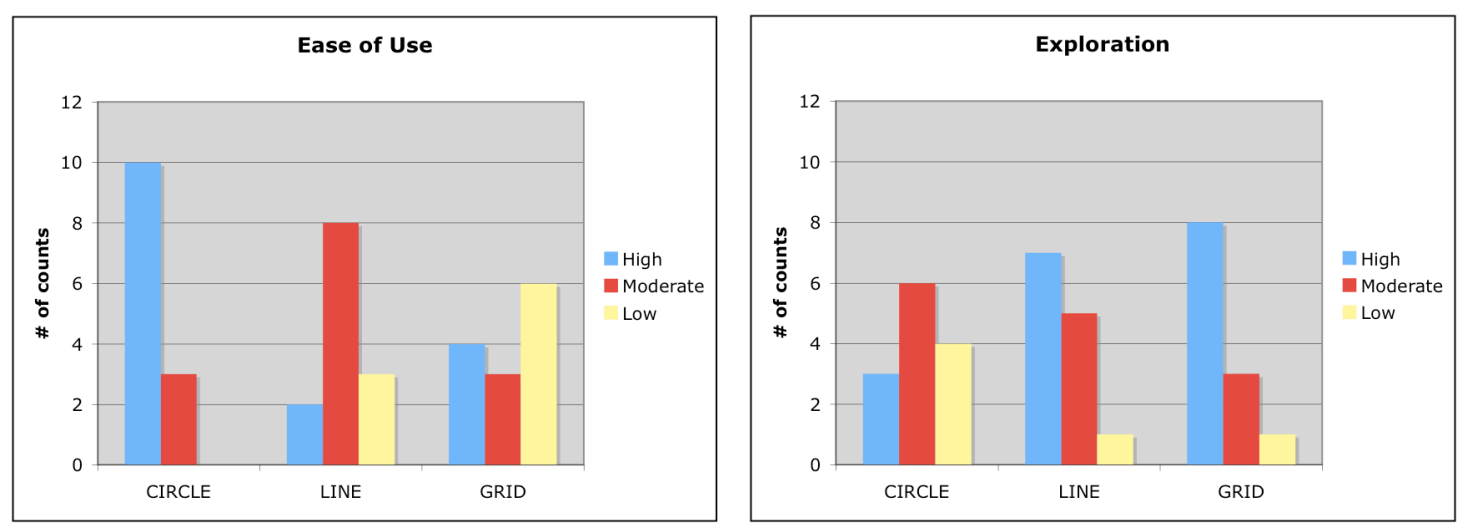

Fig 5: Responses to test models, ranking matrix graphs for Ease of Use (left) and Exploration. 
Subsequent to these preferences, the log sheets of observations were examined to calculate the duration each participant took to complete the exploration phase and the two tasks for each test model (Fig 6).

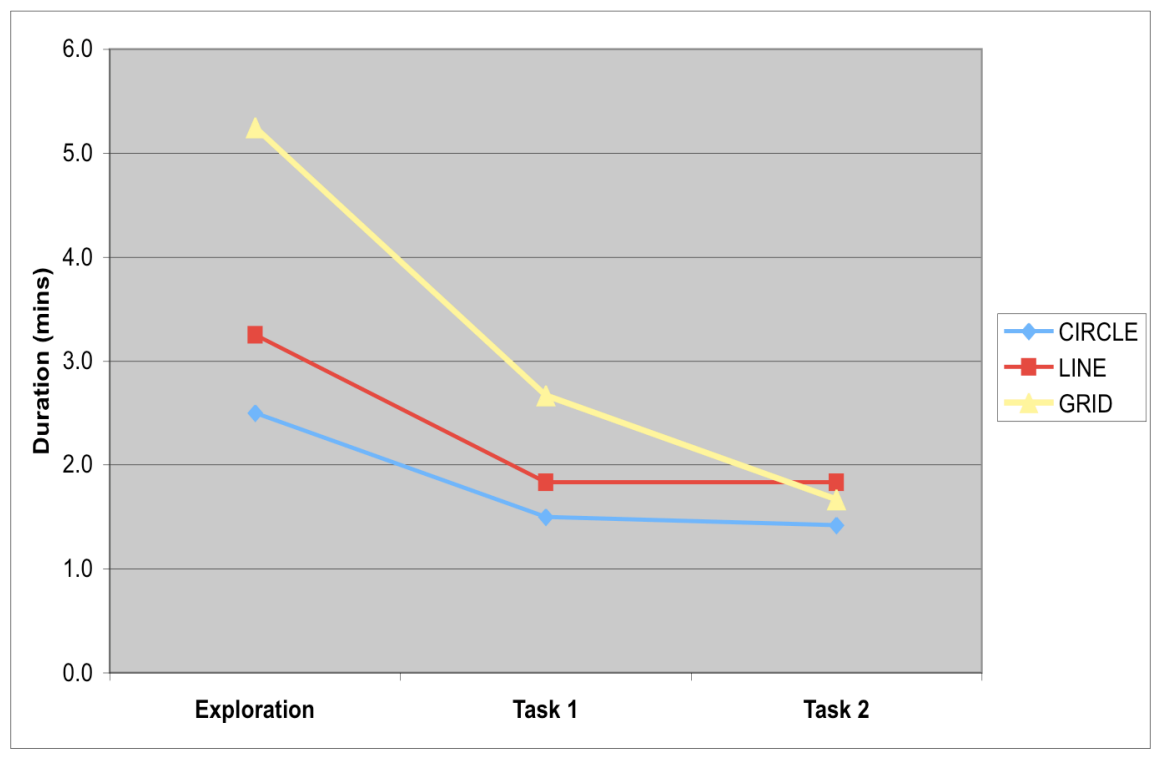

Fig 6: Average durations for exploration; task 1; and task 2.

The Figure 6 shows that the learning gained during exploration had an effect in task completion within all models and relatively more significant effect within the GRID model. The reason for this difference might be two fold: 1) the GRID model involved more complex orientation (turning left or right on the streets) and therefore the development of a greater number of spatial skills was required; 2) the GRID model required a greater amount of mnemonic effort, as the streets look alike, making it harder for the user to remember specific Locations. The Figure 6 also shows that participants spent more time exploring the GRID model compared to CIRCLE and LINE models, the reason being to acquire the skill sets required for confident navigation. Task 1 completion time was significantly higher in GRID while Task 2 completion time is similar to LINE and CIRCLE models. This implies that once users become familiar with the structure of the GRID model they perform as quickly as they did in the other two models. 


\subsection{Analysis and the emergence of Personas}

Initial analysis followed the plan proposed at the outset, based upon the three types of user profiles: expert, movie-maker, movie-user. During the process of conducting evaluation studies, revising and adjusting methods employed as the data is examined in detail may become necessary. As Preece has observed: "Most observational data is qualitative and analysis often involves interpreting what users were doing or saying by looking for patterns in the data." (Preece et al., 2002), 379. We looked for patterns visible in the graphs (Figure 5) based on the ranking data gathered from the second questionnaire. The user preferences suggested that they regarded each of the different models served for different needs and purposes. Further analysis of behavioural data gathered from the testing sessions demonstrated two different 'styles' of interaction were observed. One interaction style was task completion aiming to be quick, structured and efficient. The other style was exploration oriented to be more leisurely and engaging. The data showed the two styles were spread across the three previously described user profile groups. These two interaction behaviours led to the emergence of two personas: Quickies - those who regarded the system as a tool for finding stuff; and Explorers - those who expressed a preference for spending time experiencing the system.

Persona development has been used in user-centred design studies, and has proved to help interaction designers in making decisions about design features and functionalities (Cooper, 1999, Leritz-Higgins, 2004, Pruitt, 2003). Personas utilize our mind's powerful ability to extrapolate from partial knowledge of people to create coherent wholes and project them into new settings and situations related to an activity, in this case, working with movies on a computer. In this study we utilized personas to represent specific interaction behaviours whilst using the Mnemovie system. This is different from the way personas are employed in UCD, where personas are created to drive design; in our study personas emerge from the evaluation. Our two personas both need a storage and retrieval system for movie files that meets their practical needs whilst also complimenting their personalities. Meet, Jacky Flash (Quickie) and James Kirk (Explorer). (In text box below, commencing paragraph is an imaginary scenario or 
context extrapolated from the data. Preferences are expressed beneath in quotations, based on actual words and expressions used by Quickie and Explorer participants.)

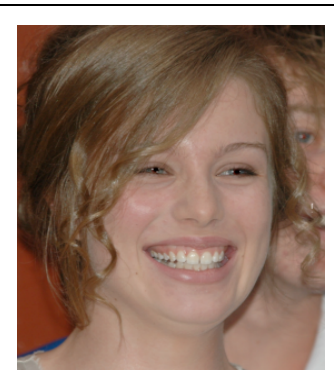

Quickie Persona - Jacky Flash

Jackie works in the ICT industry and has amassed a

large collection of home movie files shot on her mobile

phone. These she stores on her home computer and

needs to show her family when they visit, as her way of

illustrating what she has been doing, who she has been seeing, etc:

How Jackie uses Mnemovie:

"I like the interaction that enables me to accelerate the movie from one location to another, triggering search functions so that I am able to go faster, over there, there, there..... Whilst I understand the object is about locating content, my preference is to quickly tab through many movies as I talk. Like many of my friends, I quickly develop my own principle of interaction, consistently using and finding the most direct method for finding what I want...”

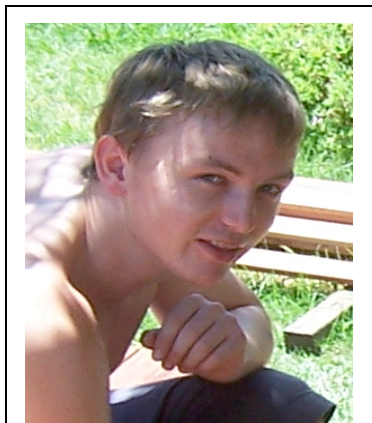

\section{Explorer Persona - James Kirk}

James is a photographer and keen local

environmentalist. He uses his video camera to record the places and people who are the subject of the issues for which he campaigns. He organises collected material on his computer so he can 'perform' his research findings, a bit like a lecture but with his subjects and their stories centre-stage.

How James uses Mnemovie:

"I intuitively interact with the system using visual cues related to the ecosystem I'm discussing, and my memory of which part of a movie can enable me to find the next movie. Each time it's like an intuitive sense of discovery. I 
learn to 'know' where a movie is, without really consciously knowing, enjoying the mystery of it and the challenge..... I patiently learn what can happen. It gives me lots of ideas too about the extent to which I can vary the content of the stories, because it is itself exploratory. I feel this is interacting with video rather than a computer."

Using these personas as a lens with which to review the data, we find that Explorers spent significantly more time exploring the GRID and CIRCLE models compared to time spent exploring the LINE model. Explorers spent significantly more time to complete Task 1 in the GRID model compared to all other conditions. Quickies spent significantly more time to Explore and complete Task 1 within the GRID model, compared to time spent interacting within the LINE and CIRCLE models. This shows that all participants whether they are Quickies or Explorers spent more time to explore and learn the GRID model. Time to complete Task 2 with the GRID model was similar to completing Task 2 with other models. This shows that participants' performances were similar to complete a task once they explored and learned how to navigate within the model. When we look at performances within the same model, we observed that Explorers always spent more time to explore and complete Task 1 compared to the Quickies. This behaviour aligns with our persona development, that there is a certain tendency for interaction style amongst participants.

This supports the conclusions drawn by Leritz-Higgins that question the user classification approach, (Leritz-Higgins, 2004) as conflating the participant's skill set and domain knowledge. As the current User Profile is based on participant's usage of movie files and their degree of experience in doing so, the evaluation was modified to include employing 'personas' to reveal conclusions useful for informing further design. 


\subsection{Discussion}

Whilst the user profile approach was a useful starting point for this evaluation, its lack of clarity (as critiqued by Leritz-Higgins) has nonetheless contributed to understanding a user experience as being much less about user skills with specific interaction designs and more about user styles of interaction. The styles of the two personas and interaction with the models can be characterised as follows:

Quickies: require an outcome with efficiencies aimed at retrieving specific files, using an interface layout and interaction devices that are functional and consistent, providing a direct equivalence between the images or the text in each video file.

Explorers: require an outcome emphasising the experience, part of which is the participant having to deduce initially the characteristics of the collection and the syntax employed in relating a moment in the motion picture with a linked movie file.

Design approaches aimed at a specific outcome - an immersive experience or a functional tool is unlikely to be as simple as, in the words of one of the participants, ' ..whether you want it to be more exploratory or more findy/searchy'. For instance, the data showed that the majority of participants were Highly Confident in interacting with the CIRCLE model where there was a clearly related visual relation between the 'index' movie and the linked movies. While most participants were Confident with the GRID model, only some appreciated its more complex interactive basis. As one observed: "...it is much more available to the ruminative and poetic mode of knowing than the instrumentalist mode of knowing..." Nonetheless the GRID model led several to describe scenarios for applying the model to their own video collections. For one with an extensive collection of feature films on a file server, using the schema could turn selecting the movie to be watched into an preliminary expedition: "In this street, my thriller movie collection; in this one, the comedies; in this house all the Marx Bros; in this dark garage at the back, my horror movies." The evaluation of the Mnemovie hypervideo interactive design at this point in the development process, turned to further reflection on the experimental models 
developed at the outset, as having significant implications useful to participants as designers of personal hypervideo systems. In the same way as the process of evaluation has informed our plans for subsequent interactive experiences and scenarios based on movie file collections, the value of openness to experimentation and reflection by the users of creativity support tools needs to be encouraged at early stages.

\section{Implications for Interaction Design}

With the advent of the personal computer design and creative authoring as practice, of text documents predominantly, has become widespread. The design concept for one of the participant's feature film collection described above, does not present a problem for the collection owner but the availability of tools capable of hypervideo design and authoring does. Whilst the microprocessor technology has also delivered the handycam, a tool most people can use with confidence, organising the material (using non-linear editing software tools) is limited to imitating the linear, sequential conventions of cinema and television. Authoring of motion picture files as hypervideo documents will require a different generation of creativity support tools.

The design approach of such tools will need to respond to the needs of those with movie file collections and the expectations of those who will access them. For Quickies and Explorers, a hyper-video system could have alternative interactive schemas, created by the collector from the support tool and selected by the viewer according to preference or need (Fig7).

\begin{tabular}{|l|l|l|}
\hline $\begin{array}{l}\text { Interactive } \\
\text { Experience }\end{array}$ & \multicolumn{2}{|c|}{ Personas Design Qualities } \\
\hline & Quickies & Explorers \\
\hline Mnemonics & $\begin{array}{l}\text { Familiar cultural } \\
\text { coding (text; actors) }\end{array}$ & $\begin{array}{l}\text { Subtle coding } \\
\text { (discovered; intuited) }\end{array}$ \\
\hline Interactive options & Sparse & Rich \\
\hline Navigational options & Less & More \\
\hline Movie speed & Faster & Slower \\
\hline
\end{tabular}

Fig 7: interactive experiences as design qualities 
The explorative viewer, in being able to select the style of interaction preferred for interacting with a collection of movie files, would address the value of the experience. In the words of one of the participants, 'I valued the maker crafting a path, resulting in a satisfying experience.' Another participant '... was totally surprised at how different the beginning and end experiences were ... just navigating the same corpus [collection].' The Quickie experience as one of the participants described, would be more '...like reading a book ... like jumping links on the internet really ... something to do by yourself as your mind goes from one track to another...'. This project has concentrated on a computer literate group, revealing the disparity between those who are task orientated and those who de-emphasise the task in favour of the experience. Hypervideo authoring will need to account for a wide range of strategies in the design of tools capable of responding to creative solutions. Though such a tool would ideally seek to reduce the additional word annotation associated with text-based systems, the participant studies suggest a toolset will necessarily incorporate text-based indexing. The kind of mnemonics and visual cueing investigated here, that are implicit and relational to a linked movie, will also require further research.

In the traditional UCD process, initial user research defines the design problem so that the designer, in correctly framing the problem can come up with a solution. In order to gather enough information for the concept, user interviews, analysis of context, patterns of use and consumption, and development of personas, are often the initial stage for interaction design. The outcome of the concept development is a design that involves implementation.

The practitioner was actively engaged in all user research activities such as selecting and recruiting participants, observing their interactions with the system, establishing criteria for the measure of their engagement and their expressed preferences. The in-depth interviews increased the practitioner's awareness of user's experience and understanding of the system. The evaluation study brought interaction design methods into the creative process through an understanding of the lived-experiences of participants. 


\section{Conclusion}

The process of designing an interactive system is complex and needs feedback at early stages of conceptual design and prototyping. The research we conducted combined several methods to capture different dimensions of user experience. We aimed at achieving an evaluation design balancing efficiency, engagement and creativity support to highlight different experience levels amongst a test group of digital movie users. Inconsistency found in the initial analysis of the data suggested to us that participants regarded each of the different models tested as being effective for different purposes. Subsequent analysis revealed significant difference in styles of interaction among participants. This led to the emergence of Quickie and Explorer personas as a means of informing subsequent user evaluations and interaction designs for hyper-video systems.

Hypervideo document design strategies for motion picture collections from all manner of sources - feature films to mobile phone grabs - will need to be further researched across a wider user base. Hypervideo systems designed for trans-lingual contexts and domains in which knowledge classification is explicit, for instance zoology, geology etc., could be of particular interest. New research will explore more effective and affecting means by which creators and audiences can store and retrieve the video files with which we work, communicate and entertain ourselves.

\section{References}

AMIR, A. P., D. BLANCHARD, B. PETKOVIC, D. SRINIVASAN, S. COHEN G. (2000.) Using Audio Time Scale Modification for Video Browsing. Proceedings of the 33rd Hawaii International Conference on System Sciences.

BALLARD, D. \& BROWN, C. (1982) Computer Vision, New Jersey, Prentice-Hall.

BILDA, Z. (2006) Evaluating Audience Experience with Interactive Art. Engage Symposium Interaction, Art \& Audience Experience. Sydney, Creativity \& Cognition Studios Press.

CANDY, L. (2007) New Media Arts and the Future of Technologies IN Creativity Support Tools. Communications of the ACM, 50, 30-31.

COOPER, A. (1999) The Inmates are Running the Asylum, Hemel Hempstead, Prentice Hall.

DAVENPORT, G. (1996) Indexes Are Out. Visions \& Views. MIT Media Lab.

GIRGENSOHN, A., SHIPMAN, F. \& WILCOX, L. (2003) Hyper-Hitchcock: Authoring Interactive Videos and Generating Interactive Summaries. $M M^{\prime} 03$. Berkeley, Ca., ACM. 
KARAT, C. M. (1994) A Comparison of user interface Evaluation methods. IN NIELSEN, J. \& MACK, R. L. (Eds.) Usability Inspection Methods. New York, John Wiley \& Sons.

LERITZ-HIGGINS, S. (2004) Practicing Persona Development: an In-House Case Study. Usability and Information Design. Society for Technical Communications.

LIM, S., SMITH, R. \& LU, G. (2004) i-Map: an interactive visualisation and navigation system of an image database for finding a sample image to initiate a visual query. OZCHI. Melbourne, Monash University.

PREECE, J., ROGERS, Y. \& SHARP, H. (2002) Interaction Design - beyond human-computer interaction, NYC, John Wiley.

PRUITT, J. (2003) Personas: Practice and theory. Proceedings of the 2003 Conference on Designing for User Experiences, 6th - 7th June, 2003. San Francisco, California, ACM. 